hep-th/0403200

\title{
Energy Momentum Tensor and Marginal Deformations in Open String Field Theory
}

\author{
Ashoke Sen \\ Harish-Chandra Research Institute \\ Chhatnag Road, Jhusi, Allahabad 211019, INDIA \\ E-mail: ashoke.sen@cern.ch, sen@mri.ernet.in
}

\begin{abstract}
Marginal boundary deformations in a two dimensional conformal field theory correspond to a family of classical solutions of the equations of motion of open string field theory. In this paper we develop a systematic method for relating the parameter labelling the marginal boundary deformation in the conformal field theory to the parameter labelling the classical solution in open string field theory. This is done by first constructing the energy-momentum tensor associated with the classical solution in open string field theory using Noether method, and then comparing this to the answer obtained in the conformal field theory by analysing the boundary state. We also use this method to demonstrate that in open string field theory the tachyon lump solution on a circle of radius larger than one has vanishing pressure along the circle direction, as is expected for a codimension one D-brane.
\end{abstract}




\section{Contents}

1 Introduction and Summary $\quad 2$

2 Energy Momentum Tensor of Non-local Field Theory 4

3 Application to $p$-adic String Theory 13

4 Application to Lump Solutions in String Field Theory 15

5 Marginal Deformation in Open String Field Theory 16

\section{Introduction and Summary}

Methods of boundary conformal field theory have been used extensively to construct classical solutions in open string field theory. This exploits the correspondence between solutions of the equations of motion of open string field theory and conformally invariant boundary interactions in two dimenional conformal field theories. One particularly important class of solutions obtained this way are those associated with marginal boundary deformations of the conformal field theory $[1,2,3,4,5]$. Such deformations typically generate a continuous family of conformal field theories, and hence one expects that they will generate a continuous family of solutions of the open string field equations.

An algorithm for constructing such a family of solutions in open string field theory was developed in $[6,7,8]$ using the level truncation method $[9,10,11,12]$. This involves choosing an arbitrary value of the open string field associated with the marginal operator,

solving the equations of motion of all other fields, and substituting them back into the action. This generates an effective potential for the first field. It was demonstrated numerically that as we increase the level of approximation, the effective potential becomes flatter. This suggests that in the full theory this effective potential is exactly flat, and hence we have a one parameter family of solutions labelled by the value of the open string field associated with the marginal operator.

This algorithm gives a way to generate a one parameter family of solutions in open string field theory if the corresponding conformal field theory has a marginal operator. However one of the problems which was not resolved in [6] was to give an algorithm to systematically determine the relation between the marginal deformation parameter in 
the conformal field theory and the parameter labelling the solutions in open string field theory. This is the problem we address in this paper. We work in the context of the specific class of marginal deformations analyzed in ref.[6], namely deformation by the first momentum mode of the tachyon on a D-brane in bosonic string theory wrapped on a circle of unit radius. Our main tool is to compare the energy-momentum tensor of the system in the two descriptions. In the conformal field theory description, the information about the energy-momentum tensor is contained in the boundary state[13]. For the case at hand the exact expression for the boundary state of the system is known as a function of the marginal deformation parameter $[1,3]$ and using this one can compute the energy-momentum tensor of the system. In particular the pressure along the compact direction, given by the diagonal component of the stress tensor along this direction, has a simple expression in terms of the deformation parameter. We compare this with the corresponding result for the solution of the open string field equations. Matching the two results determine the relationship between the parameter labelling the solutions of open string field theory and that labelling the boundary conformal field theories.

In order to implement this algorithm we need to construct the energy momentum tensor $T_{\rho}^{\mu}$ of open string field theory[14] which contains non-local interactions. Energymomentum tensor of non-local field theories was previously analyzed in $[15,16,17,18]$, but we derive a different form of $T_{\rho}^{\mu}$ which is suitable for our analysis. We test our final expression by applying it to the usual scalar field theory, as well as to the $p$-adic string theory and reproducing the known answers up to the usual ambiguity of defining energymomentum tensor using Noether prescription. We then apply it to open string field theory to compute the appropriate component of the $T_{\rho}^{\mu}$ associated with the family of solutions found in [6], and compare the results with the conformal field theory results to derive the relation between the conformal field theory parameter and string field theory parameter.

The paper is organised as follows. In section 2 we derive a form of the energymomentum tensor for a general translationally invarint non-local field theory. In section 3 we use this to compute the energy of a time dependent classical solution in $p$-adic string theory and reproduce the answer given in [15]. In section 4 we use the expression for $T_{\nu}^{\mu}$ derived in section 2 to compute the pressure associated with the lump solution in open string field theory on a D-string wrapped on a circle of radius $>1$, and show that it vanishes. This agress with the identification of the lump solution as the D0-brane located at a point on the circle, which is known to have vanishing pressure along the circle direction. 
This provides another consistency check for our formula. Finally in section 5 we apply our result to calculate the pressure associated with the family of lump solutions in open string field theory wrapped on a circle of unit radius. Comparing this with the conformal field theory results, we then numerically determine the relation between the parameter labelling this family of solutions and the parameter labelling the corresponding conformal field theories.

Although in this paper we shall be working in the specific context of bosonic string theory, the methods developed here are equally applicable to the analysis of classical solutions in open superstring field theory on unstable D-p-branes.

\section{Energy Momentum Tensor of Non-local Field The- ory}

We begin by reviewing the general procedure for constructing a conserved current associated with a continuous symmetry. Let us consider a theory with scalar fields $\phi_{1}, \phi_{2}, \ldots$ in $p+1$ dimensions, and let $S\left[\left\{\phi_{r}\right\}\right]$ be a functional of these fields describing the action. Now suppose that $S$ is invariant under an infinitesimal symmetry transformation of the form:

$$
\delta \phi_{r}(x)=\epsilon f_{r}\left[\left\{\phi_{s}\right\} ; x\right],
$$

where $f_{r}\left[\left\{\phi_{s}\right\} ; x\right]$ is a functional of the fields $\left\{\phi_{s}\right\}$ and a function of $x$, and $\epsilon$ is an infinitesimal parameter. Under this transformation $\delta S=0$.

Now consider a modified transformation law

$$
\delta \phi_{r}(x)=\epsilon(x) f_{r}\left[\left\{\phi_{s}\right\} ; x\right]
$$

The only difference between (2.1) and (2.2) is that in the former $\epsilon$ is a constant whereas in the latter we have taken $\epsilon$ to be space-time dependent. In general (2.2) is not a symmetry of $S$. Hence $\delta S$ does not vanish. However it must be proportional to $\partial_{\mu} \epsilon$ since it should vanish whenever $\epsilon$ is set to a constant. The general expression for $\delta S$ will thus have the form

$$
\delta S=-\int d^{p+1} x \partial_{\mu} \epsilon J^{\mu}(x)=\int d^{p+1} x \epsilon \partial_{\mu} J^{\mu}(x),
$$

where $J^{\mu}(x)$ is a function of $x$ and functional of the fields $\left\{\phi_{s}\right\}$. Eq.(2.3) defines the currents $J^{\mu}(x)$ up to addition of a term $K^{\mu}$ which satisfies $\partial_{\mu} K^{\mu}=0$ without using 
equations of motion. This is the usual ambiguity in the construction of conserved currents using Noether prescription.

So far we have not used equations of motion. If the functions $\phi_{r}$ happen to be solutions of the equation of motion derived from the action $S$, then for any variation $\delta \phi_{r}$ of $\phi_{r}$ (including the ones given in (2.2)) $\delta S$ must vanish to first order in $\epsilon$. Thus in this case we must have

$$
\partial_{\mu} J^{\mu}(x)=0 .
$$

In other words the currents $J^{\mu}(x)$ defined through (2.3) are conserved when the fields satisfy their equations of motion. This is Noether's theorem.

Since we shall be dealing with theories with non-local action, it is best to work in the momentum space. If we define by $\widetilde{\phi}_{r}(k), \widetilde{J}^{\mu}(k)$ and $\widetilde{\epsilon}(k)$ the Fourier transforms of $\phi_{r}(x)$, $J^{\mu}(x)$ and $\epsilon(x)$ :

$\widetilde{\phi}_{r}(k)=\int d^{p+1} x e^{-i k \cdot x} \phi_{r}(x), \quad \widetilde{J}^{\mu}(k)=\int d^{p+1} x e^{-i k \cdot x} J^{\mu}(x), \quad \widetilde{\epsilon}(k)=\int d^{p+1} x e^{-i k \cdot x} \epsilon(x)$,

then (2.3) takes the form:

$$
\delta S=i \int \frac{d^{p+1} q}{(2 \pi)^{p+1}} \epsilon(-q) q_{\mu} \widetilde{J}^{\mu}(q) .
$$

We shall now use the definition of $J^{\mu}(x)$ given in (2.3) to construct the energymomentum tensor $T_{\rho}^{\mu}$ of a classical field theory with non-local action. $T_{\rho}^{\mu}$ is the conserved current associated with the translational symmetry:

$$
\delta \phi_{r}(x)=\epsilon \partial_{\rho} \phi_{r}(x) .
$$

In order to find $T_{\rho}^{\mu}$ we consider the modified transformation:

$$
\delta \phi_{r}(x)=\epsilon(x) \partial_{\rho} \phi_{r}(x)
$$

or in the momentum space,

$$
\delta \widetilde{\phi}_{r}(k)=i \int \frac{d^{p+1} q}{(2 \pi)^{p+1}} \epsilon(-q)\left(k_{\rho}+q_{\rho}\right) \widetilde{\phi}_{r}(k+q) .
$$

Then $\widetilde{T}_{\rho}^{\mu}$ is defined by expressing $\delta S$ under the transformation (2.9) as

$$
\delta S=i \int \frac{d^{p+1} q}{(2 \pi)^{p+1}} \epsilon(-q) q_{\mu} \widetilde{T}_{\rho}^{\mu}(q) .
$$


Of course this equation does not necessarily fix $T_{\rho}^{\mu}$ uniquely. We can add terms of the form $K_{\rho}^{\mu}$ to $T_{\rho}^{\mu}$ if $\partial_{\mu} K_{\rho}^{\mu}=0$ without using equations of motion. Normally this ambiguity does not affect the definition of the total energy and total momentum if the field configuration is regular so that boundary terms from spatial infinity can be ignored during integration by parts.

In order to proceed further, we need to begin with some general form of $S$. We express $S$ as a power series expansion in the fields $\phi_{r}$ :

$$
\begin{gathered}
S=\sum_{N \geq 2} \int \frac{d^{p+1} k_{1}}{(2 \pi)^{p+1}} \int \frac{d^{p+1} k_{2}}{(2 \pi)^{p+1}} \ldots \int \frac{d^{p+1} k_{N-1}}{(2 \pi)^{p+1}} A_{r_{1} \cdots r_{N}}^{(N)}\left(k_{1}, \ldots k_{N-1}\right) \\
\phi_{r_{1}}\left(k_{1}\right) \cdots \phi_{r_{N-1}}\left(k_{N-1}\right) \phi_{r_{N}}\left(-k_{1}-\ldots-k_{N-1}\right) .
\end{gathered}
$$

Then, under (2.9), we have

$$
\begin{aligned}
\delta S= & i \sum_{N \geq 2} \int \frac{d^{p+1} q}{(2 \pi)^{p+1}} \epsilon(-q) \int \frac{d^{p+1} k_{1}}{(2 \pi)^{p+1}} \int \frac{d^{p+1} k_{2}}{(2 \pi)^{p+1}} \ldots \int \frac{d^{p+1} k_{N-1}}{(2 \pi)^{p+1}} \\
& {\left[k_{1 \rho}\left\{A_{r_{1} \cdots r_{N}}^{(N)}\left(k_{1}-q, \ldots k_{N-1}\right)-A_{r_{1} \cdots r_{N}}^{(N)}\left(k_{1}, \ldots, k_{N-1}\right)\right\}+\cdots\right.} \\
& +k_{(N-1) \rho}\left\{A_{r_{1} \cdots r_{N}}^{(N)}\left(k_{1}, \ldots, k_{N-1}-q\right)-A_{r_{1} \cdots r_{N}}^{(N)}\left(k_{1}, \ldots k_{N-1}\right)\right\} \\
& \left.+q_{\rho} A_{r_{1} \cdots r_{N}}^{(N)}\left(k_{1}, \ldots k_{N-1}\right)\right] \phi_{r_{1}}\left(k_{1}\right) \cdots \phi_{r_{N-1}}\left(k_{N-1}\right) \phi_{r_{N}}\left(-k_{1}-\ldots-k_{N-1}+q\right) \\
\equiv & i \int \frac{d^{p+1} q}{(2 \pi)^{p+1}} \epsilon(-q) q_{\mu} \widetilde{T}_{\rho}^{\mu}(q) .
\end{aligned}
$$

This gives an expression for $q_{\mu} \widetilde{T}_{\rho}^{\mu}$. From this we can extract $\widetilde{T}_{\rho}^{\mu}$ up to the usual ambiguity. A possible prescription, which we shall adopt, is to expand the terms involving $A_{r_{1} \ldots r_{N}}^{(N)}$ (but not those involving the $\phi_{r}$ 's) in Taylor series expansion in $q$, and identify the coefficient of $q_{\mu}$ coming from this expansion in a Lorentz covariant fashion. This gives

$$
\begin{aligned}
\widetilde{T}_{\rho}^{\mu}(q)= & \sum_{N=2}^{\infty} \int \prod_{j=1}^{N-1} \frac{d^{p+1} k_{j}}{(2 \pi)^{p+1}}\left[\delta_{\rho}^{\mu} A_{r_{1} \ldots r_{N}}^{(N)}\left(k_{1}, \ldots k_{N-1}\right)\right. \\
& \left.+\sum_{i=1}^{N-1} \sum_{s=0}^{\infty} k_{i \rho}(-1)^{s+1} \frac{1}{(s+1) !} \frac{\partial^{s+1} A_{r_{1} \ldots r_{N}}^{(N)}\left(k_{1}, \ldots k_{N-1}\right)}{\partial k_{i \mu} \partial k_{i \mu_{1}} \ldots \partial k_{i \mu_{s}}} q_{\mu_{1}} \ldots q_{\mu_{s}}\right] \\
& \phi_{r_{1}}\left(k_{1}\right) \cdots \phi_{r_{N-1}}\left(k_{N-1}\right) \phi_{r_{N}}\left(-k_{1}-\ldots-k_{N-1}+q\right) .
\end{aligned}
$$

It is easy to verify that $q_{\mu} \widetilde{T}_{\rho}^{\mu}$ for $\widetilde{T}_{\rho}^{\mu}$ given in (2.13) satisfies $(2.12)$. 
(2.13) gives the form of $\widetilde{T}_{\rho}^{\mu}$ in the general case. It is instructive to ensure that this gives the correct $T_{\rho}^{\mu}$ for a free scalar field $\phi$ of mass $m$. In this case only $A^{(2)}\left(k_{1}\right)$ is non-zero and has the value $\frac{1}{2}\left(-k_{1}^{2}-m^{2}\right)$. Substituting this into (2.13) and Fourier transforming the resulting expression we recover the correct $T_{\rho}^{\mu}$ for this theory up to the addition of a term proportional to $\left(\partial^{\mu} \partial_{\rho}-\delta_{\rho}^{\mu} \square\right) \phi^{2}$. This additional term is conserved without using equations of motion and reflects the ambiguity in the definition of $T_{\rho}^{\mu}$ using Noether prescription. This term does not affect the definition of total energy and total momentum of the system as long as $\phi$ is well behaved at $\infty$.

If we are interested in the total energy-momentum vector $P_{\rho}\left(x^{0}\right)=\int d^{p} x T_{\rho}^{0}(x)$, then their Fourier transform in time $-\widetilde{P}_{\rho}\left(q^{0}\right)$ - is given by $\left.\widetilde{T}_{\rho}^{0}(q)\right|_{\vec{q}=0} \cdot(2.13)$ gives:

$$
\begin{aligned}
\widetilde{P}_{\rho}\left(q^{0}\right)= & \sum_{N=2}^{\infty} \prod_{j=1}^{N-1} \frac{d^{p+1} k_{j}}{(2 \pi)^{p+1}}\left[\delta_{\rho}^{0} A_{r_{1} \ldots r_{N}}^{(N)}\left(k_{1}, \ldots k_{N-1}\right)\right. \\
& \left.+\sum_{i=1}^{N-1} \frac{k_{i \rho}}{q^{0}}\left(A_{r_{1} \ldots r_{N}}^{(N)}\left(k_{1}, \ldots k_{i}-q, \ldots k_{N-1}\right)-A_{r_{1} \ldots r_{N}}^{(N)}\left(k_{1}, \ldots k_{N-1}\right)\right)\right] \\
& \left.\phi_{r_{1}}\left(k_{1}\right) \cdots \phi_{r_{N-1}}\left(k_{N-1}\right) \phi_{r_{N}}\left(-k_{1}-\ldots-k_{N-1}+q\right)\right|_{\vec{q}=0} .
\end{aligned}
$$

We shall now use (2.13) to derive the form of $T_{\nu}^{\mu}$ for the special case where the field configuration depends on only one of the space-time coordiates $x$. We shall take $x$ to be a Euclidean coordinate, but the case where $x$ is the time coordinate can easily be derived from this by a double Wick rotation. ${ }^{1}$ In momentum space we can express $\widetilde{\phi}_{r}(k)$ as:

$$
\widetilde{\phi}_{r}(k)=(2 \pi)^{p} \tilde{\chi}_{r}\left(k_{x}\right) \prod_{\sigma \neq x} \delta\left(k_{\sigma}\right)
$$

where $k_{x}$ is the momentum conjugate to $x$. First consider the case $\rho \neq x$ in (2.13). In this case the contribution to the terms in (2.13) involving $k_{i \rho}$ vanish due to the delta functions in the $\widetilde{\phi}_{r}(k)$. Thus we have

$$
\begin{gathered}
\widetilde{T}_{\rho}^{\mu}(q)=(2 \pi)^{p} \delta_{\rho}^{\mu} \prod_{\sigma \neq x} \delta\left(q_{\sigma}\right) \sum_{N \geq 2} \int^{N-1} \prod_{i=1}^{N-1} \frac{d k_{i x}}{2 \pi} A_{r_{1} \cdots r_{N}}^{(N)}\left(k_{1 x}, \ldots k_{(N-1) x}\right) \\
\tilde{\chi}_{r_{1}}\left(k_{1 x}\right) \cdots \widetilde{\chi}_{r_{N-1}}\left(k_{(N-1) x}\right) \tilde{\chi}_{r_{N}}\left(-k_{1 x}-\ldots-k_{(N-1) x}+q_{x}\right), \\
\text { for } \rho \neq x .
\end{gathered}
$$

\footnotetext{
${ }^{1}$ In this case $T_{\rho}^{\mu}$ gives the energy-momentum tensor of a spatially homogeneous time dependent background.
} 
Let us also assume that the field configuration has a discrete space-time symmetry such that if we define

$$
A_{r_{1} \cdots r_{N} ;(i, \sigma)}^{(N)}\left(k_{1}, \ldots k_{N-1}\right) \equiv \frac{\partial}{\partial k_{i \sigma}} A_{r_{1} \cdots r_{N}}^{(N)}\left(k_{1}, \ldots k_{N-1}\right),
$$

where $k_{i \sigma}$ denotes the component of the $i$ 'th momentum $k_{i}$ along the $\sigma$ direction, then for any set $\left(r_{1}, \ldots r_{N}\right)$ for which $\phi_{r_{i}}$ 's do not vanish, we have

$$
A_{r_{1} \cdots r_{N} ;(i, \sigma)}^{(N)}\left(k_{1}, \ldots k_{N-1}\right)=0 \quad \text { if } k_{j \sigma}=0 \text { for every } j .
$$

Note that in evaluating the right hand side of (2.17) we first need to differentiate with respect to $k_{i \sigma}$ for a general set of momenta and then set $k_{j \sigma}$ to zero for every $j$ in (2.18). Using (2.13), (2.15) and (2.18) we can see that in this case

$$
T_{x}^{\mu}=0 \quad \text { for } \mu \neq x .
$$

This leaves us to determine $T_{x}^{x}$. Due to the factors of $\prod_{\sigma \neq x} \delta\left(k_{\sigma}\right)$ in the expression for $\phi_{r}(k)$ we see that in (2.13) we shall get a factor proportional to $\prod_{\sigma \neq x} \delta\left(q_{\sigma}\right)$, and hence in this expression for $T_{x}^{x}$ all the indices $\mu, \mu_{1}, \ldots \mu_{s}$ can only be equal to $x$. In this case we can resum the series expansion to express $\widetilde{T}_{x}^{x}$ as

$$
\begin{aligned}
\widetilde{T}_{x}^{x}(q)= & (2 \pi)^{p} \prod_{\sigma \neq x} \delta\left(q_{\sigma}\right) \sum_{N \geq 2} \int \prod_{i=1}^{N-1} \frac{d k_{i x}}{2 \pi}\left[A_{r_{1} \cdots r_{N}}^{(N)}\left(k_{1 x}, \ldots k_{(N-1) x}\right)\right. \\
& \left.+\sum_{i=1}^{N-1} k_{i x} \frac{1}{q_{x}}\left\{A_{r_{1} \cdots r_{N}}^{(N)}\left(k_{1 x}, \ldots k_{i x}-q_{x}, \ldots k_{(N-1) x}\right)-A_{r_{1} \cdots r_{N}}^{(N)}\left(k_{1 x}, \ldots k_{(N-1) x}\right)\right\}\right] \\
& \tilde{\chi}_{r_{1}}\left(k_{1 x}\right) \cdots \tilde{\chi}_{r_{N-1}}\left(k_{(N-1) x}\right) \tilde{\chi}_{r_{N}}\left(-k_{1 x}-\ldots-k_{(N-1) x}+q_{x}\right) .
\end{aligned}
$$

The ambiguity in determining $\widetilde{T}_{\rho}^{\mu}(q)$ using Noether prescription corresponds to adding to $\widetilde{T}_{\rho}^{\mu}(q)$ terms of the form $\widetilde{K}_{\rho}^{\mu}(q)=\left(q^{\mu} q_{\rho}-q^{2} \delta_{\rho}^{\mu}\right) F(q)$ for some $F(q)$. For field configurations of the form we are considering $\widetilde{K}_{\rho}^{\mu}(q)$ has support in the subspace of the $q$-space in which all components of $q$ other than the $x$-component are set to zero. Hence $\widetilde{K}_{x}^{x}=0$, and $\widetilde{T}_{x}^{x}(q)$ is unambiguously defined in this case.

So far we have not assumed that the field configuration $\tilde{\chi}_{r}\left(q_{x}\right)$ satisfies its equation of motion. However we shall be interested in computing $T_{\nu}^{\mu}$ only for those configurations which satisfy equations of motion. In this case due to the conservation law $\partial_{\mu} T_{\rho}^{\mu}=0$, 
and that $T_{x}^{\mu}=0$ for $\mu \neq x, T_{x}^{x}$ must be $x$ independent. This information can be used to derive various different formulæ for $T_{x}^{x}$ all of which are physically invariant. The most straightforward formula for $T_{x}^{x}$ is obtained by taking the Fourier transform of $\widetilde{T}_{x}^{x}(q)$ :

$$
\begin{aligned}
T_{x}^{x}(x)= & \int \prod_{\sigma} \frac{d q_{\sigma}}{2 \pi} e^{i q_{\sigma} x^{\sigma}} \widetilde{T}_{x}^{x}(q) \\
= & \sum_{N \geq 2} \int \frac{d q_{x}}{2 \pi} e^{i q_{x} x} \prod_{i=1}^{N-1} \frac{d k_{i x}}{2 \pi}\left[A_{r_{1} \cdots r_{N}}^{(N)}\left(k_{1 x}, \ldots k_{(N-1) x}\right)\right. \\
& \left.+\sum_{j=1}^{N-1} k_{j x} \frac{1}{q_{x}}\left\{A_{r_{1} \cdots r_{N}}^{(N)}\left(k_{1 x}, \ldots k_{j x}-q_{x}, \ldots k_{(N-1) x}\right)-A_{r_{1} \cdots r_{N}}^{(N)}\left(k_{1 x}, \ldots k_{(N-1) x}\right)\right\}\right] \\
& \widetilde{\chi}_{r_{1}}\left(k_{1 x}\right) \cdots \widetilde{\chi}_{r_{N-1}}\left(k_{(N-1) x}\right) \tilde{\chi}_{r_{N}}\left(-k_{1 x}-\ldots-k_{(N-1) x}+q_{x}\right) .
\end{aligned}
$$

For application to string field theory, a different formula will be useful. Since $T_{x}^{x}$ is independent of $x, \widetilde{T}_{x}^{x}(q)$ must be proportional to $\delta\left(q_{x}\right)$. The non-trivial information about $T_{x}^{x}$ is then contained in the coefficient of the $\delta\left(q_{x}\right)$ in $\widetilde{T}_{x}^{x}(q)$. In order to extract this information we need to evaluate (2.20) near $q_{x}=0$, setting $q_{x}=0$ in all terms which have smooth $q_{x} \rightarrow 0$ limit. Since we shall only consider theories for which the coefficients $A_{r_{1} \cdots r_{N}}^{(N)}\left(k_{1 x}, \ldots k_{(N-1) x}\right)$ are smooth functions of their arguments, we get $^{2}$

$$
\begin{aligned}
\widetilde{T}_{x}^{x}\left(q_{x}\right)= & (2 \pi)^{p} \prod_{\sigma \neq x} \delta\left(q_{\sigma}\right) \sum_{N \geq 2} \int \prod_{i=1}^{N-1} \frac{d k_{i x}}{2 \pi}\left[A_{r_{1} \cdots r_{N}}^{(N)}\left(k_{1 x}, \ldots k_{(N-1) x}\right)\right. \\
& \left.-\sum_{j=1}^{N-1} k_{j x} A_{r_{1} \cdots r_{N} ;(j, x)}^{(N)}\left(k_{1 x}, \ldots k_{(N-1) x}\right)\right] \\
& \widetilde{\chi}_{r_{1}}\left(k_{1 x}\right) \cdots \widetilde{\chi}_{r_{N-1}}\left(k_{(N-1) x}\right) \tilde{\chi}_{r_{N}}\left(-k_{1 x}-\ldots-k_{(N-1) x}+q_{x}\right),
\end{aligned}
$$

where $A_{r_{1} \cdots r_{N} ;(i, x)}^{(N)}\left(k_{1 x}, \ldots k_{(N-1) x}\right)$ have been defined in $(2.17)$. Note that we have not set $q_{x}=0$ inside the argument of $\widetilde{\chi}_{r_{N}}$ in anticipation of the fact $\tilde{\chi}_{r}\left(k_{x}\right)$ may not be a smooth function of $k_{x}$. In fact if the $\tilde{\chi}_{r}\left(k_{x}\right)$ are smooth functions (which is the case if $\chi_{r}(x)$ falls off to zero as $\left.x \rightarrow \pm \infty\right)$ then there is no possibility of getting a delta function singularity as $q_{x} \rightarrow 0$ in $(2.22)$ and hence $T_{x}^{x}$ must vanish. This is expected. Since $T_{x}^{x}$

\footnotetext{
${ }^{2}$ Although this expression for $T_{x}^{x}$ looks quite different from the one given in [15] for $x$ dependent field configurations, the expression given in [15] can be brought to the form given in (2.22) for on-shell field configurations by manipulations involving space-averaging, integration by parts and resummation.
} 
must be independent of the coordinate $x$, we can evaluate it at $x=\infty$, and hence for a field configuration which vanishes as $x \rightarrow \infty T_{x}^{x}$ must also vanish.

In order to construct solutions with non-trivial $T_{x}^{x}$, we can consider periodic field configurations along $x$ direction. If the period is $2 \pi R$ then $\chi_{r}(x)$ must have the form:

$$
\chi_{r}(x)=\sum_{n=-\infty}^{\infty} \tilde{\chi}_{r, n} e^{i n x / R}
$$

This gives

$$
\tilde{\chi}_{r}\left(q_{x}\right)=\int d x e^{-i q_{x} x} \chi_{r}(x)=2 \pi \sum_{n=-\infty}^{\infty} \tilde{\chi}_{r, n} \delta\left(q_{x}-\frac{n}{R}\right) .
$$

Substituting this into (2.22) we get near $q_{x}=0:^{3}$

$$
\begin{aligned}
\widetilde{T}_{x}^{x}\left(q_{x}\right)= & (2 \pi)^{p+1} \prod_{\sigma} \delta\left(q_{\sigma}\right) \sum_{N \geq 2} \sum_{n_{1}, \ldots n_{N-1}} \tilde{\chi}_{r_{1}, n_{1}} \ldots \tilde{\chi}_{r_{N-1}, n_{N-1}} \tilde{\chi}_{r_{N},-n_{1}-\ldots-n_{N-1}} \\
& {\left[A_{r_{1}, \ldots r_{N}}^{(N)}\left(\frac{n_{1}}{R}, \ldots \frac{n_{N-1}}{R}\right)-\sum_{i=1}^{N-1} \frac{n_{i}}{R} A_{r_{1}, \ldots r_{N} ;(i, x)}^{(N)}\left(\frac{n_{1}}{R}, \ldots \frac{n_{N-1}}{R}\right)\right] . }
\end{aligned}
$$

After taking Fourier transform this gives

$$
\begin{aligned}
T_{x}^{x}= & \sum_{N \geq 2} \sum_{n_{1}, \ldots n_{N-1}} \tilde{\chi}_{r_{1}, n_{1}} \ldots \tilde{\chi}_{r_{N-1}, n_{N-1}} \tilde{\chi}_{r_{N},-n_{1}-\ldots-n_{N-1}} \\
& {\left[A_{r_{1}, \ldots r_{N}}^{(N)}\left(\frac{n_{1}}{R}, \ldots \frac{n_{N-1}}{R}\right)-\sum_{i=1}^{N-1} \frac{n_{i}}{R} A_{r_{1}, \ldots r_{N} ;(i, x)}^{(N)}\left(\frac{n_{1}}{R}, \ldots \frac{n_{N-1}}{R}\right)\right] . }
\end{aligned}
$$

For completeness we shall also write down the expression for $T_{\rho}^{\mu}$ for $\rho \neq x$ in this case. Using (2.16) and (2.24) we get

$$
\begin{aligned}
\widetilde{T}_{\rho}^{\mu}(q)= & (2 \pi)^{p} \delta_{\rho}^{\mu} \prod_{\sigma \neq x} \delta\left(q_{\sigma}\right) \sum_{N \geq 2} \sum_{n_{1}, \ldots n_{N}} A_{r_{1}, \ldots r_{N}}^{(N)}\left(\frac{n_{1}}{R}, \ldots \frac{n_{N-1}}{R}\right) \\
& \widetilde{\chi}_{r_{1}, n_{1}} \ldots \tilde{\chi}_{r_{N-1}, n_{N-1}} \tilde{\chi}_{r_{N}, n_{N}} 2 \pi \delta\left(q_{x}-\frac{1}{R} \sum_{i=1}^{N} n_{i}\right), \quad \text { for } \rho \neq x .
\end{aligned}
$$

\footnotetext{
${ }^{3}$ By the general argument leading to the conservation law of $T_{\rho}^{\mu}$, the contribution to $\widetilde{T}_{x}^{x}(n / R)$ for $n \neq 0$ vanishes if all the $\widetilde{\chi}_{r, n}$ satisfy their equations of motion.
} 
Taking its Fourier transform gives:

$$
\begin{aligned}
T_{\rho}^{\mu}(x)= & \delta_{\rho}^{\mu} \sum_{N \geq 2} \sum_{n_{1}, \ldots n_{N}} \exp \left(i \frac{x}{R} \sum_{i=1}^{N} n_{i}\right) A_{r_{1}, \ldots r_{N}}^{(N)}\left(\frac{n_{1}}{R}, \ldots \frac{n_{N-1}}{R}\right) \\
& \tilde{\chi}_{r_{1}, n_{1}} \ldots \widetilde{\chi}_{r_{N-1}, n_{N-1}} \tilde{\chi}_{r_{N}, n_{N}}, \quad \text { for } \rho \neq x .
\end{aligned}
$$

The average $T_{\rho}^{\mu}$ over a period is given by:

$$
\left\langle T_{\rho}^{\mu}\right\rangle=\delta_{\rho}^{\mu} \sum_{N \geq 2} \sum_{n_{1}, \ldots n_{N-1}} A_{r_{1}, \ldots r_{N}}^{(N)}\left(\frac{n_{1}}{R}, \ldots \frac{n_{N-1}}{R}\right) \tilde{\chi}_{r_{1}, n_{1}} \ldots \widetilde{\chi}_{r_{N-1}, n_{N-1}} \widetilde{\chi}_{r_{N},-n_{1}-\ldots-n_{N-1}}
$$

During the analysis described above, we have given a special role to the $N^{\prime}$ th momentum $k_{N}$ by replacing it by $-\left(k_{1}+\ldots k_{N-1}\right)$ and representing $A_{r_{1} \ldots r_{N}}^{(N)}$ as a function of the momenta $k_{1}, \ldots k_{N-1}$. For practical computation it is often convenient ${ }^{4}$ to use all the $N$ arguments $k_{1}, \ldots k_{N}$ by expressing $A_{r_{1} \ldots r_{N}}^{(N)}$ as a function $\mathcal{A}_{r_{1}, \ldots r_{N}}^{(N)}$ of $k_{1}, \ldots k_{N}$. (This of course is not unique since we can replace the whole or part of the $k_{N}$ by $\left.-\left(k_{1}+\ldots+k_{N-1}\right)\right)$. The results in one formalism can be transformed to the other formalism by using the equation:

$$
\frac{\partial}{\partial k_{i x}} A_{r_{1} \cdots r_{N}}^{(N)}\left(k_{1 x}, \ldots k_{(N-1) x}\right)=\frac{\partial}{\partial k_{i x}} \mathcal{A}_{r_{1} \cdots r_{N}}^{(N)}\left(k_{1 x}, \ldots k_{N x}\right)-\frac{\partial}{\partial k_{N x}} \mathcal{A}_{r_{1} \cdots r_{N}}^{(N)}\left(k_{1 x}, \ldots k_{N x}\right),
$$

where it is understood that on both the right and the left hand side we take the partial derivative keeping all the other arguments fixed. This gives:

$$
\sum_{i=1}^{N-1} k_{i x} \frac{\partial}{\partial k_{i x}} A_{r_{1} \cdots r_{N}}^{(N)}\left(k_{1 x}, \ldots k_{(N-1) x}\right)=\sum_{i=1}^{N} k_{i x} \frac{\partial}{\partial k_{i x}} \mathcal{A}_{r_{1} \cdots r_{N}}^{(N)}\left(k_{1 x}, \ldots k_{N x}\right),
$$

since $k_{N x}=-\left(k_{1 x}+\ldots+k_{(N-1) x}\right)$. Thus if we define

$$
\mathcal{A}_{r_{1} \cdots r_{N} ;(i, x)}^{(N)}\left(k_{1 x}, \ldots k_{N x}\right) \equiv \frac{\partial}{\partial k_{i x}} \mathcal{A}_{r_{1} \cdots r_{N}}^{(N)}\left(k_{1 x}, \ldots k_{N x}\right)
$$

we can rewrite $(2.22)$ and $(2.26)$ as

$$
\widetilde{T}_{x}^{x}\left(q_{x}\right)=(2 \pi)^{p} \prod_{\sigma \neq x} \delta\left(q_{\sigma}\right) \sum_{N \geq 2} \int \prod_{i=1}^{N} \frac{d k_{i x}}{2 \pi} \quad 2 \pi \delta\left(k_{1 x}+\ldots k_{N x}-q_{x}\right)
$$

\footnotetext{
${ }^{4}$ For example, if the indices $r_{1}, \ldots r_{N}$ take the same value, then it is more convenient to regard $A_{r_{1} \ldots r_{N}}^{(N)}$ as a symmetric function of $k_{1}, \ldots k_{N}$.
} 


$$
\begin{aligned}
& {\left[\mathcal{A}_{r_{1} \cdots r_{N}}^{(N)}\left(k_{1 x}, \ldots k_{N x}\right)-\sum_{j=1}^{N} k_{j x} \mathcal{A}_{r_{1} \cdots r_{N} ;(j, x)}^{(N)}\left(k_{1 x}, \ldots k_{N x}\right)\right]} \\
& \tilde{\chi}_{r_{1}}\left(k_{1 x}\right) \cdots \widetilde{\chi}_{r_{N 1}}\left(k_{N x}\right),
\end{aligned}
$$

and

$$
\begin{aligned}
T_{x}^{x}= & \sum_{N \geq 2} \sum_{n_{1}, \ldots n_{N}} \delta_{n_{1}+\ldots n_{N}, 0} \widetilde{\chi}_{r_{1}, n_{1}} \ldots \widetilde{\chi}_{r_{N}, n_{N}} \\
& {\left[\mathcal{A}_{r_{1}, \ldots r_{N}}^{(N)}\left(\frac{n_{1}}{R}, \ldots \frac{n_{N}}{R}\right)-\sum_{i=1}^{N} \frac{n_{i}}{R} \mathcal{A}_{r_{1}, \ldots r_{N} ;(i, x)}^{(N)}\left(\frac{n_{1}}{R}, \ldots \frac{n_{N}}{R}\right)\right], }
\end{aligned}
$$

respectively. Also, eq.(2.29) takes the form

$$
\left\langle T_{\rho}^{\mu}\right\rangle=\delta_{\rho}^{\mu} \sum_{N \geq 2} \sum_{n_{1}, \ldots n_{N}} \mathcal{A}_{r_{1}, \ldots r_{N}}^{(N)}\left(\frac{n_{1}}{R}, \ldots \frac{n_{N}}{R}\right) \tilde{\chi}_{r_{1}, n_{1}} \ldots \widetilde{\chi}_{r_{N}, n_{N}} \delta_{n_{1}+\ldots n_{N}, 0} \quad \text { for } \rho \neq x
$$

These formulæ look manifestly symmetric in all the arguments.

We can further simplify (2.34), (2.35) as follows. Using (2.11), (2.15) and (2.24) we can express the action $S$ as

$$
S\left(\left\{\chi_{r, n}\right\} ; R\right)=V_{p+1} \mathcal{L}_{a v}\left(\left\{\chi_{r, n}\right\} ; R\right),
$$

where $V_{p+1} \equiv(2 \pi)^{p+1} \delta^{p+1}(k=0)$ denotes the total volume of the D-brane world-volume, and

$$
\mathcal{L}_{a v}\left(\left\{\chi_{r, n}\right\} ; R\right)=\sum_{N \geq 2} \sum_{n_{1}, \ldots n_{N}} \mathcal{A}_{r_{1}, \ldots r_{N} ;(i, x)}^{(N)}\left(\frac{n_{1}}{R}, \ldots \frac{n_{N}}{R}\right) \widetilde{\chi}_{r_{1}, n_{1}} \ldots \widetilde{\chi}_{r_{N}, n_{N}} \delta_{n_{1}+\ldots n_{N}, 0},
$$

is the space-time averaged Lagrangian density. Thus we see using (2.35) that

$$
\left\langle T_{\rho}^{\mu}\right\rangle=\delta_{\rho}^{\mu} \mathcal{L}_{a v} \quad \text { for } \rho \neq x .
$$

Also, using (2.32) we get

$$
\sum_{i=1}^{N} \frac{n_{i}}{R} \mathcal{A}_{r_{1}, \ldots r_{N} ;(i, x)}^{(N)}\left(\frac{n_{1}}{R}, \ldots \frac{n_{N}}{R}\right)=-R \frac{\partial}{\partial R} \mathcal{A}_{r_{1}, \ldots, r_{N}}^{(N)}\left(\frac{n_{1}}{R}, \ldots \frac{n_{N}}{R}\right) .
$$

Using eqs.(2.37), (2.39) we can express (2.34) as

$$
T_{x}^{x}=\mathcal{L}_{a v}+R \frac{\partial \mathcal{L}_{a v}}{\partial R}=\frac{\partial}{\partial R}\left(R \mathcal{L}_{a v}\right),
$$


where in $(2.40)$ the derivative with respect to $R$ has to be computed for fixed $\tilde{\chi}_{r, n}$ 's.

In some situations the theory may contain a family of solutions, with the periodicity $2 \pi R$ of the solution varying continuously inside the family. Thus we have a family of solutions labelled by $R$. In this case we can define $\mathcal{L}_{a v}$ to be a function of $R$ alone by replacing $\widetilde{\chi}_{r, n}$ by their classical solutions, and evaluating the expression for $\mathcal{L}_{a v}$. The total derivative $\frac{d \mathcal{L}_{a v}}{d R}$ of $\mathcal{L}_{a v}$ will now include contribution due to explicit $R$ dependence of the coefficients $\mathcal{A}_{r_{1}, \ldots r_{N} ;(i, x)}^{(N)}\left(\frac{n_{1}}{R}, \ldots \frac{n_{N}}{R}\right)$ appearing in $\mathcal{L}_{a v}$ as well as due to the $R$ dependence of the solutions $\tilde{\chi}_{r, n}$. However, since $\left\{\tilde{\chi}_{s, m}\right\}$ are solutions of the equations of motion, the derivative of $\mathcal{L}_{a v}$ with respect to $\tilde{\chi}_{r, n}$ vanishes for each $(r, n)$ and $\frac{d \mathcal{L}_{a v}}{d R}$ is actually equal to $\frac{\partial \mathcal{L}_{a v}}{\partial R}$ appearing in (2.40). Thus in this case we can replace $\frac{\partial}{\partial R}$ in $(2.40)$ by total derivative

$\frac{d}{d R}$. Since from (2.38) we see that $2 \pi R \mathcal{L}_{a v}$ can be interpreted as the integral of $T_{0}^{0}$ over a period, if we regard $x$ as a compact direction with period $2 \pi R$ then $-2 \pi R \mathcal{L}_{a v}$ has the interpretation of the total energy of the solution. Hence $\frac{d}{d R}\left(R \mathcal{L}_{a v}\right)$ has the interpretation of being the negative of the derivative of the total energy with respect to the period. This precisely coincides with the usual definition of pressure of any system.

\section{Application to $p$-adic String Theory}

In this section we shall apply the results of section 2 to derive the form of the stress tensor in $l$-adic string theory and compare with the results of $[15,16]$. We begin with the action of the $l$-adic string theory $[19,20,21]$ :

$$
S=\int d^{p+1} x \mathcal{L}, \quad \mathcal{L}=\frac{1}{g_{l}^{2}}\left[-\frac{1}{2} \phi l^{-\frac{1}{2} \square} \phi+\frac{1}{l+1} \phi^{l+1}\right],
$$

where $l$ is an integer, $\phi$ is a scalar field and $g_{l}$ is the coupling constant of the open $l$-adic string theory. Comparing this with eq.(2.11) we see that in this case:

$$
\begin{array}{ll}
A^{(2)}\left(k_{1}\right)=-\frac{1}{2 g_{l}^{2}} l^{\frac{1}{2} k_{1}^{2}}, \quad & A^{(l+1)}\left(k_{1}, \ldots k_{l}\right)=\frac{1}{g_{l}^{2}} \frac{1}{l+1}, \\
A_{r_{1} \ldots r_{N}}^{(N)}\left(k_{1}, \ldots k_{N-1}\right)=0 & \text { for } N \neq 2, l+1 .
\end{array}
$$

For the case of odd $l$, ref.[15] constructed a one parameter family of time dependent solution of the form:

$$
\phi\left(x^{0}\right)=\sum_{n=0}^{\infty} a_{2 n+1} \cos \left((2 n+1) \omega x^{0}\right)
$$


labelled by the arbitrary constant $\omega$. For any given $\omega$ the coefficients $a_{2 n+1}$ are determined from the equations of motion. We shall now find an expression for $T_{0}^{0}$ for such a field configuration using the general formalism given in section 2 and compare with the corresponding expression obtained in [15].

We derived various formulæ for $T_{x}^{x}$ for an $x$-dependent field configuration in section 2. We can take any of these and make an inverse Wick rotation $x \rightarrow-i x^{0}$ to get the answer for $T_{0}^{0}$ for a time dependent field configuration. The formula that directly yields the result of [15] is eq.(2.21) at $x=0$, giving the expresstion for $T_{0}^{0}$ at $x^{0}=0$ after inverse Wick rotation. The first term inside the square bracket on the right hand side of (2.21) just gives the lagrangian density $\mathcal{L}$ evaluated at $x^{0}=0$. Since the other terms involve differences in $A^{(N)}$ evaluated for two different momentum arguments, and since from (3.2) we see that $A^{(l+1)}$ does not depend on momenta, this term receives contribution only for $N=2$. Using (3.1)-(3.3), (2.21) takes the form:

$T_{0}^{0}=\mathcal{L}\left(x^{0}=0\right)-\frac{1}{2 g_{l}^{2}} \sum_{m, n \geq 0} a_{2 m+1} a_{2 n+1} \frac{(2 m+1)^{2}}{(2 m+1)^{2}-(2 n+1)^{2}}\left(l^{-\frac{1}{2}(2 n+1)^{2} \omega^{2}}-l^{-\frac{1}{2}(2 m+1)^{2} \omega^{2}}\right)$.

Note that the $m=n$ term in the above expression has $0 / 0$ form and hence must be defined as the result of taking $m \rightarrow n$ limit. This gives a modified expression for (3.4)

$$
\begin{aligned}
T_{0}^{0}= & \mathcal{L}\left(x^{0}=0\right)-\frac{1}{4 g_{l}^{2}} \omega^{2} \ln l \sum_{n=0}^{\infty} a_{2 n+1}^{2}(2 n+1)^{2} l^{-\frac{1}{2}(2 n+1)^{2} \omega^{2}} \\
& -\frac{1}{2 g_{l}^{2}} \sum_{\substack{m, n \geq 0 \\
m \neq n}} a_{2 m+1} a_{2 n+1} \frac{(2 m+1)^{2}}{(2 m+1)^{2}-(2 n+1)^{2}}\left(l^{-\frac{1}{2}(2 n+1)^{2} \omega^{2}}-l^{-\frac{1}{2}(2 m+1)^{2} \omega^{2}}\right) .
\end{aligned}
$$

This agrees with the result derived in [15]. One simplicity of our starting formula (2.21) is that we arrive at the answer (3.5) without having to do any resummation as in [15]. Using the equations of motion $\phi^{l}=l^{-\frac{1}{2} \square} \phi$, and (3.1), (3.3) we can write down a simple expression for $\mathcal{L}\left(x^{0}=0\right)$ appearing in (3.5):

$$
\mathcal{L}\left(x^{0}=0\right)=\frac{1}{2 g_{l}^{2}} \frac{1-l}{1+l} \sum_{m, n \geq 0} a_{2 m+1} a_{2 n+1} l^{-\frac{1}{2} \omega^{2}(2 n+1)^{2}} .
$$




\section{Application to Lump Solutions in String Field The- ory}

In this section we shall apply the results of section 2 to calculate $T_{x}^{x}$ associated with the lump solutions in open string field theory $[22,23]$. As in [22], we shall focus on a D-string of bosonic string theory with the direction $x$ tangential to the D-string compactified on a circle of radius $R$. The action of the open string field theory on the D-string is given by:

$$
S=-\frac{1}{g_{o}^{2}}\left[\frac{1}{2}\left\langle\Phi\left|Q_{B}\right| \Phi\right\rangle+\frac{1}{3}\langle\Phi \mid \Phi * \Phi\rangle\right],
$$

where $|\Phi\rangle$ is the string field represented by a ghost number 1 state in the Hilbert space of the first quantized string theory, * denotes the usual open string star product[14] and $g_{o}$ is the open string coupling constant. In [22] the space averaged lagrangian density associated with a time independent, periodic field configuration was denoted by:

$$
\mathcal{L}_{a v}(|\Phi\rangle ; R)=-2 \pi^{2} \mathcal{T}_{1} \mathcal{V}(|\Phi\rangle ; R)
$$

Unlike in the case of ref.[22] here we have explicitly displayed the $R$ dependence of $\mathcal{V}$. Let us denote by $\left|\Phi_{l u m p}\right\rangle$ the lump solution in this string field theory, representing a D0-brane localized at $x=0$. Using (2.40) we can express $T_{x}^{x}$ associated with this solution as:

$$
T_{x}^{x}\left(\left|\Phi_{\text {lump }}\right\rangle\right)=-2 \pi^{2} \mathcal{T}_{1}\left(\mathcal{V}\left(\left|\Phi_{\text {lump }}\right\rangle ; R\right)+R \mathcal{V}^{\prime}\left(\left|\Phi_{\text {lump }}\right\rangle ; R\right)\right)
$$

where

$$
\mathcal{V}^{\prime}(|\Phi\rangle ; R) \equiv \frac{\partial \mathcal{V}(|\Phi\rangle ; R)}{\partial R}
$$

with $\partial / \partial R$ denoting derivative with respect to $R$ at fixed $|\Phi\rangle$. Since the original D-string has $T_{x}^{x}=-\mathcal{T}_{1}$, and since the final D0-brane should have vanishing $T_{x}^{x}$, we see that the $T_{x}^{x}\left(\left|\Phi_{\text {lump }}\right\rangle\right)$ computed from the string field theory must cancel the $T_{x}^{x}$ of the D-string. This leads to the conjecture:

$$
T_{x}^{x}\left(\left|\Phi_{\text {lump }}\right\rangle\right)=\mathcal{T}_{1}
$$

Using (4.3) this takes the form:

$$
-2 \pi^{2}\left(\mathcal{V}\left(\left|\Phi_{\text {lump }}\right\rangle ; R\right)+R \mathcal{V}^{\prime}\left(\left|\Phi_{\text {lump }}\right\rangle ; R\right)\right)=1
$$

On the other hand from (2.38) we see that the $T_{0}^{0}$ associated with the solution is equal to the average lagrangian density $-2 \pi^{2} \mathcal{T}_{1} \mathcal{V}\left(\left|\Phi_{\text {lump }}\right\rangle ; R\right)$. Thus the total energy associated 
with the lump solution, obtained by multiplying $-T_{0}^{0}$ by $2 \pi R$, and adding to it the energy $2 \pi R \mathcal{T}_{1}$ of the original D-string, will be given by[22]:

$$
2 \pi R \mathcal{T}_{1}\left(2 \pi^{2} \mathcal{V}\left(\left|\Phi_{\text {lump }}\right\rangle ; R\right)+1\right)
$$

If $\left|\Phi_{\text {lump }}\right\rangle$ has to describe a D0-brane then its energy must be equal to the expected mass of the D0-brane which is $2 \pi \mathcal{T}_{1}$. Thus the energy conjecture implies[22]

$$
R\left(2 \pi^{2} \mathcal{V}\left(\left|\Phi_{\text {lump }}\right\rangle ; R\right)+1\right)=1
$$

(4.8) was verified numerically in [22], and using the expression of $\mathcal{V}(|\Phi\rangle ; R)$ and the numerical solution for $\left|\Phi_{\text {lump }}\right\rangle$ given there we can also verify (4.6) numerically. But we shall now show that the pressure conjecture (4.6) follows automatically from the energy conjecture (4.8). For this we differentiate (4.8) with respect to $R$ to get

$$
2 \pi^{2}\left(\mathcal{V}\left(\left|\Phi_{\text {lump }}\right\rangle ; R\right)+R \frac{d \mathcal{V}\left(\left|\Phi_{\text {lump }}\right\rangle ; R\right)}{d R}\right)+1=0,
$$

where the derivative with respect to $R$ now also acts on the various $R$ dependent coefficients appearing in the expansion of $\left|\Phi_{\text {lump }}\right\rangle$. However, using the argument at the end of section 2 we can replace $d \mathcal{V}\left(\left|\Phi_{\text {lump }}\right\rangle ; R\right) / d R$ by $\partial \mathcal{V}\left(\left|\Phi_{\text {lump }}\right\rangle ; R\right) / \partial R=\mathcal{V}^{\prime}\left(\left|\Phi_{\text {lump }}\right\rangle ; R\right)$ where the $R$ derivative acts only on the coefficients appearing in $\mathcal{V}((|\Phi\rangle ; R)$ at fixed $|\Phi\rangle$. Eq.(4.9) then reduces to (4.6).

Thus we see that for the tachyon lump solution in open string field theory, the vanishing of the total pressure in direction transverse to the lump is not an independent conjecture, but follows from the conjecture that the total energy of the lump solution is given by the mass of the D0-brane, which in turn is independent of the radius of the circle. While this does not lead to a new test of the validity of string field theory, this shows that the formula for $T_{x}^{x}$ for a periodic solution, as given in (2.40), is correct. In section 5 we shall use (2.40) to relate the marginal deformation parameter in the conformal field theory description of a solution to the parameter labelling the corresponding solution in string field theory.

\section{Marginal Deformation in Open String Field The- ory}

In section 4 we calculated $T_{x}^{x}$ associated with the lump solution in open string field theory for a D-string on a circle of radius $R$ and showed that the result agrees with the prediction 
based on the identification of the solution as a D0-brane. In this section we shall consider the same system at $R=1$. In this case instead of having a unique lump solution there is a one parameter family of lump solutions. In the language of conformal field theory this corresponds to adding to the world-sheet action a boundary term:

$$
\lambda \int d t \cos (X(t))
$$

where $X$ is the world-sheet scalar field describing the compact coordinate, $\lambda$ is a parameter labelling the deformed conformal field theory and $t$ is a parameter labelling the boundary of the world-sheet. Total $T_{x}^{x}$ associated with this solution can be calculated from the boundary state associated with the deformed conformal field theory $[1,3]$ and takes the value $[4,5]$

$$
\left.T_{x}^{x}\right|_{C F T}=-\mathcal{T}_{1} \cos ^{2}(\pi \lambda)
$$

Since we expect a one to one correspondence between the classical solutions of open string field equations and boundary conformal field theories, we expect that the open string field theory on a D-string wrapped on a circle of unit radius must also have a one parameter family of classical solutions. A systematic procedure for constructing these classical solutions using level truncation approximation $[9,10,11,12]$ was developed in [6]. We look for a string field configuration of the form:

$$
|\Phi\rangle=\sum_{n=0}^{\infty}\left[t_{n} c_{1}+u_{n} c_{-1}+v_{n} c_{1} L_{-2}^{X}+w_{n} L_{-2}^{\prime}+\cdots\right] \cos \left(\frac{n}{R} X(0)\right)|0\rangle,
$$

subject to the Siegel gauge condition, periodicity along $x$, invariance under twist and $x \rightarrow$ $-x$ transformation etc. Here $t_{n}, u_{n}, v_{n}$ etc. are coefficients labelling a given string field configuration, $c_{n}, b_{n}$ are oscillators of the ghost fields, $L_{n}^{X}$ denote the Virasoro generators of the $c=1$ conformal field theory associated with the $X$ field, $L_{n}^{\prime}$ denote the Virasoro generators of the $c=25$ conformal field theory involving rest of the fields, and $\cdots$ stand for infinite number of other terms involving other ghost number one operators constructed out of $c_{n}, b_{n}, L_{n}^{X}$ and $L_{n}^{\prime}$.

A given classical solution in this theory corresponds to a fixed set of values for the coefficients $t_{n}, u_{n}, v_{n}$ etc. Thus a one parameter family of solutions will correspond to a one parameter family of $\left\{t_{n}, u_{n}, v_{n}, \ldots\right\}$. To leading order in $\lambda$ the solution corresponds to

$$
t_{1} \simeq \lambda
$$


with all other coefficients being zero. This suggested the following procedure for constructing the one parameter family of solutions. For a generic $R$, we first fix a value of $t_{1}$, and solve for the other coefficients by using their equations of motion. Plugging these solutions back into the action we get an effective action $-2 \pi^{2} \mathcal{V}_{\text {eff }}\left(t_{1} ; R\right)$ for the coefficient $t_{1}$. For general $R>1, \mathcal{V}_{\text {eff }}\left(t_{1} ; R\right)$ will have a local minimum at some value of $t_{1}$, and the lump solution will correspond to this minimum. However if there really exists a one parameter familty of solutions for $R=1$ then $\mathcal{V}_{\text {eff }}\left(t_{1} ; 1\right)$ should be independent of $t_{1}$, and hence must vanish since it vanishes at $t_{1}=0$. In this case the one parameter family of solutions will be labelled by $t_{1}$. It was found using the level truncation analysis that while to any finite level of approximation $\mathcal{V}_{\text {eff }}\left(t_{1} ; 1\right)$ is not flat, it does become flatter as we increase the level of approximation. This suggested that the full open string field theory does have a one parameter family of classical solutions representing the conformal field theories associated with (5.1).

One of the problems which was left unresolved in [6] was to develop a systematic scheme for relating the parameter $t_{1}$ labelling the solution in open string field theory to the conformal field theory parameter $\lambda$ appearing in eq.(5.1) beyond the leading order result (5.4). This is the problem we address in this section. ${ }^{5}$ This will be done by comparing the expression for $T_{x}^{x}$ given in (5.2) with the $T_{x}^{x}$ associated with the lump solution given in (4.3) plus the contribution $-\mathcal{T}_{1}$ of the original D-string. This gives:

$$
-\cos ^{2}(\pi \lambda)=-1-2 \pi^{2}\left(\mathcal{V}_{e f f}\left(t_{1} ; 1\right)+\mathcal{V}_{e f f}^{\prime}\left(t_{1} ; 1\right)\right),
$$

or, equivalently,

$$
\sin ^{2}(\pi \lambda)=-2 \pi^{2}\left(\mathcal{V}_{e f f}\left(t_{1} ; 1\right)+\mathcal{V}_{e f f}^{\prime}\left(t_{1} ; 1\right)\right) \equiv F\left(t_{1}\right),
$$

where $\mathcal{V}_{\text {eff }}^{\prime}\left(t_{1} ; R\right)$ denotes the result of replacing in $\mathcal{V}^{\prime}(|\Phi\rangle, R)$ all coefficients other than $t_{1}$ by solution to their equations of motion. This is equivalent to defining $\mathcal{V}_{e f f}^{\prime}\left(t_{1} ; R\right)$ as

$$
\mathcal{V}_{e f f}^{\prime}\left(t_{1} ; R\right)=\frac{\partial}{\partial R} \mathcal{V}_{e f f}\left(t_{1} ; R\right),
$$

with the derivative computed at fixed $t_{1}$. Apparently the $\partial / \partial R$ on the right hand side of eq.(5.7) receives additional contribution besides $\mathcal{V}^{\prime}(|\Phi\rangle, R)$ due to the implicit

\footnotetext{
${ }^{5} \mathrm{An}$ analytic expression for the solution was proposed in [7]. This expression is a sum of a term linear in $\lambda$, and a term quadratic in $\lambda$. Since the open string field theory action is a cubic polynomial in the string field, the energy momentum tensor calculated for this configuration will be a polynomial function of $\lambda$ of degree 6 . It is not clear how this can reproduce the $\cos ^{2}(\pi \lambda)$ factor in the expression for $T_{x}^{x}$ given in $(5.2)$.
} 
$R$-dependence of $\mathcal{V}_{\text {eff }}\left(t_{1} ; R\right)$ through the various coefficients other than $t_{1}$ which were eliminated by their equations of motion. However since in the construction of $\mathcal{V}_{\text {eff }}\left(t_{1} ; R\right)$, $\mathcal{V}(|\Phi\rangle ; R)$ is extremized with respect to these coefficients, these additional terms do not contribute, and eq.(5.7) holds.

For real $\lambda$, the left hand side of eq.(5.6) cannot exceed unity. Thus for consistency the right hand side, which is a specific expression in string field theory, must also be bounded from above by unity. We shall now argue that this is indeed the case. In particular, assuming that the energy conjecture (4.8) holds, and that $\mathcal{V}_{\text {eff }}\left(t_{1} ; 1\right)$ is independent of $t_{1}$ and hence vanishes, we shall give an analytical proof of the fact that the function $F\left(t_{1}\right)$ defined in eq. (5.6) has a maximum where it takes the value 1 , and hence $\lambda$ defined through (5.6) is real. For this let us define by $t_{1}^{(0)}(R)$ the value of $t_{1}$ that minimizes $\mathcal{V}_{\text {eff }}\left(t_{1}, R\right)$ with respect to $t_{1}$ for a generic $R$. This definition does not work for $R=1$ since there $\mathcal{V}_{\text {eff }}\left(t_{1} ; 1\right)$ is flat, but we shall define by $t_{1}^{(0)}(1)$ the limit of $t_{1}^{(0)}(R)$ as $R \rightarrow 1$. We shall now show that

1. $F\left(t_{1}\right)$ reaches a maximum at $t_{1}=t_{1}^{(0)}(1)$.

2. $F\left(t_{1}^{(0)}(1)\right)=1$.

This will establish that the maximum value $F\left(t_{1}\right)$ can take is 1 , and hence $\sin ^{2}(\pi \lambda)$ defined through (5.6) gives real $\lambda$ for all $t_{1}$. This will also show that the point $\lambda=1 / 2$ corresponds to $t_{1}=t_{1}^{(0)}(1)$, i.e. the configuration in string field theory representing a D0-brane, since for a generic $R$ the lump solution represents a D0-brane. This is the expected result. ${ }^{6}$

We begin by proving the first result. Since $\mathcal{V}_{\text {eff }}\left(t_{1} ; 1\right)$ vanishes, we get from (5.6), (5.7)

$$
\frac{d F\left(t_{1}\right)}{d t_{1}}=-2 \pi^{2} \frac{\partial \mathcal{V}_{e f f}^{\prime}\left(t_{1}, 1\right)}{\partial t_{1}}=-2 \pi^{2}\left[\frac{\partial}{\partial R}\left(\frac{\partial \mathcal{V}_{e f f}\left(t_{1} ; R\right)}{\partial t_{1}}\right)\right]_{R=1}
$$

Substituting $t_{1}=t_{1}^{(0)}(1)$ on both sides we get:

$$
\left.\frac{d F\left(t_{1}\right)}{d t_{1}}\right|_{t_{1}=t_{1}^{(0)}(1)}=-2 \pi^{2}\left[\frac{\partial}{\partial R}\left(\left.\frac{\partial \mathcal{V}_{\text {eff }}\left(t_{1} ; R\right)}{\partial t_{1}}\right|_{t_{1}=t_{1}^{(0)}(1)}\right)\right]_{R=1}
$$

\footnotetext{
${ }^{6}$ Before getting into the formal proof it is worth giving an intuitive argument. Since $\mathcal{V}_{\text {eff }}\left(t_{1} ; 1\right)$ is expected to vanish, we have $-2 \pi^{2} \mathcal{V}_{\text {eff }}\left(t_{1} ; 1+\epsilon\right)=-2 \pi^{2} \epsilon \mathcal{V}_{\text {eff }}^{\prime}\left(t_{1} ; 1\right)+\mathcal{O}\left(\epsilon^{2}\right)=\epsilon F\left(t_{1}\right)+\mathcal{O}\left(\epsilon^{2}\right)$. Thus the maximum of $F\left(t_{1}\right)$ occurs at the minimum of $\mathcal{V}_{\text {eff }}\left(t_{1} ; 1+\epsilon\right)$ for small $\epsilon$, i.e. at $t_{1}^{(0)}(1)$. The energy conjecture (4.8) tells us that $2 \pi^{2} \mathcal{V}_{\text {eff }}\left(t_{1} ; 1+\epsilon\right)=(1+\epsilon)^{-1}-1=-\epsilon+\mathcal{O}\left(\epsilon^{2}\right)$ at its minimum $t_{1}^{(0)}(1+\epsilon) \simeq t_{1}^{(0)}(1)$. This gives $F\left(t_{1}^{(0)}(1)\right)=1$.
} 


$$
=-2 \pi^{2}\left[\frac{d}{d R}\left(\left.\frac{\partial \mathcal{V}_{e f f}\left(t_{1} ; R\right)}{\partial t_{1}}\right|_{t_{1}=t_{1}^{(0)}(R)}\right)-\left(\frac{\partial^{2} \mathcal{V}_{\text {eff }}\left(t_{1} ; R\right)}{\partial t_{1}^{2}}\right)_{t_{1}=t_{1}^{(0)}(R)} \frac{d t_{1}^{(0)}(R)}{d R}\right]_{R=1}
$$

Now, by definition $\left.\left(\partial \mathcal{V}_{\text {eff }}\left(t_{1} ; R\right) / \partial t_{1}\right)\right|_{t_{1}=t_{1}^{(0)}(R)}$ vanishes for all $R$. On the other hand, at $R=1, \mathcal{V}_{\text {eff }}\left(t_{1} ; R\right)$ vanishes identically, and hence its second derivative with respect to $t_{1}$ also vanishes. Thus we see that the right hand side of (5.9) vanishes identically, and hence $F\left(t_{1}\right)$ has a extremum at $t_{1}=t_{1}^{(0)}(1)$. The fact that it is a maximum and not a minimum can be seen easily by differentiating (5.8) once more with respect to $t_{1}$ and using the fact that for $R>1, \mathcal{V}_{\text {eff }}\left(t_{1} ; R\right)$ has a minimum at $t_{1}=t_{1}^{(0)}(R)$.

It now remains to prove that $F\left(t_{1}^{(0)}(1)\right)=1$. Using eq. $(5.6)$ and the fact that $\mathcal{V}_{\text {eff }}\left(t_{1}, 1\right)$ vanishes identically we see that

$$
F\left(t_{1}^{(0)}(1)\right)=-2 \pi^{2} \mathcal{V}_{e f f}^{\prime}\left(t_{1}^{(0)}(1), 1\right)
$$

Using the energy conjecture (4.8) and that fact that $t_{1}=t_{1}^{(0)}(R)$ describes the lump solution at radius $R$, we have:

$$
R\left(2 \pi^{2} \mathcal{V}_{\text {eff }}\left(t_{1}^{(0)}(R) ; R\right)+1\right)=1
$$

Differentiating this with respect to $R$, and noting that $R$ derivative action on $t_{1}^{(0)}(R)$ can be neglected since $\mathcal{V}_{\text {eff }}\left(t_{1}, R\right)$ is extremized with respect to $t_{1}$ at $t_{1}=t_{1}^{(0)}(R)$, we get

$$
2 \pi^{2} \mathcal{V}_{e f f}\left(t_{1}^{(0)}(R) ; R\right)+1+2 \pi^{2} R \mathcal{V}_{e f f}^{\prime}\left(t_{1}^{(0)}(R) ; R\right)=0
$$

Setting $R=1$ in this equation makes the first term vanish identically. Thus the equation reduces to

$$
2 \pi^{2} \mathcal{V}_{e f f}^{\prime}\left(t_{1}^{(0)}(1) ; 1\right)=-1
$$

Hence from (5.10), (5.13) we have

$$
F\left(t_{1}^{(0)}(1)\right)=1
$$

This is the desired result.

We shall now describe the explicit computation of the function $F\left(t_{1}\right)$ using level truncation approximation. Before getting into the numerical results it will be instructive to illustrate the computation of $F\left(t_{1}\right)$ using (5.6) in a simple approximation where analytic 
solution is possible. This is level $\left(1 / R^{2}, 2 / R^{2}\right)$ approximation. ${ }^{7}$ In this approximation the only modes to be included are $t_{0}$ and $t_{1}$, and $\mathcal{V}(|\Phi\rangle ; R)$ is given by:

$$
\mathcal{V}(|\Phi\rangle ; R)=-\frac{1}{2} t_{0}^{2}-\frac{1}{4}\left(1-\frac{1}{R^{2}}\right) t_{1}^{2}+\frac{1}{3} K^{3} t_{0}^{3}+\frac{1}{2} K^{3-\frac{2}{R^{2}}} t_{0} t_{1}^{2},
$$

where

$$
K=\frac{3 \sqrt{3}}{4}
$$

This gives

$$
R \mathcal{V}^{\prime}(|\Phi\rangle ; R)=-\frac{1}{2} \frac{1}{R^{2}} t_{1}^{2}+\frac{1}{2} \frac{4}{R^{2}} \ln K K^{3-\frac{2}{R^{2}}} t_{0} t_{1}^{2}
$$

The equation $\partial \mathcal{V} / \partial t_{0}=0$ has two solutions for $t_{0}$ :

$$
t_{0}=\frac{1}{2 K^{3}}\left(1 \pm \sqrt{1-2 K^{6-\frac{2}{R^{2}}} t_{1}^{2}}\right)
$$

Of these the solution with the + sign has the property that $t_{0}$ does not vanish even when $t_{1}=0$. This branch was called the vacuum branch in [6] (since for $t_{1}=0$ it describes the tachyon vacuum solution) and is not the branch of interest to us. The other branch, called the marginal branch, gives the solution we are looking for. Thus we take

$$
t_{0}=\frac{1}{2 K^{3}}\left(1-\sqrt{1-2 K^{6-\frac{2}{R^{2}}} t_{1}^{2}}\right) .
$$

For this solution $t_{0}$ vanishes at $t_{1}=0$. Substituting this back into (5.15) and (5.17) we get $\mathcal{V}_{\text {eff }}\left(t_{1} ; R\right)$ and $R \mathcal{V}_{\text {eff }}^{\prime}\left(t_{1} ; R\right)$ respectively. This $\mathcal{V}_{\text {eff }}\left(t_{1} ; R\right)$ of course is not flat, but for $R=1$ it approaches a flat potential as we increase the level of approximation[6]. Note also that real solution for $t_{0}$ exists only for $\left|t_{1}\right| \leq 1 / \sqrt{2} K^{3-R^{-2}}$. At $\left|t_{1}\right|=1 / \sqrt{2} K^{3-R^{-2}}$ the vacuum branch and the marginal branch meet. This feature survives even at higher level of approximation so that we always get a finite range of $t_{1}$ over which the solution exists[6].

We now use eq. (5.6), (5.15) and (5.17) to find $F\left(t_{1}\right)$ and hence the relation between $\lambda$ and $t_{1}$. We get

$$
\begin{aligned}
F\left(t_{1}\right) & =-2 \pi^{2}\left[-\frac{1}{2} t_{0}^{2}-\frac{1}{4}\left(1+\frac{1}{R^{2}}\right) t_{1}^{2}+\frac{1}{3} K^{3} t_{0}^{3}+\frac{1}{2}\left(1+\frac{4}{R^{2}} \ln K\right) K^{3-\frac{2}{R^{2}}} t_{0} t_{1}^{2}\right]_{R=1} \\
& =2 \pi^{2}\left[\frac{1}{2} t_{0}^{2}+\frac{1}{2} t_{1}^{2}-\frac{1}{3} K^{3} t_{0}^{3}-\frac{1}{2}(1+4 \ln K) K t_{0} t_{1}^{2}\right]
\end{aligned}
$$

\footnotetext{
${ }^{7}$ Note that although eventually we shall set $R=1$, we must keep $R$ arbitrary, calculate $\partial \mathcal{V} / \partial R$, and only then set $R=1$.
} 


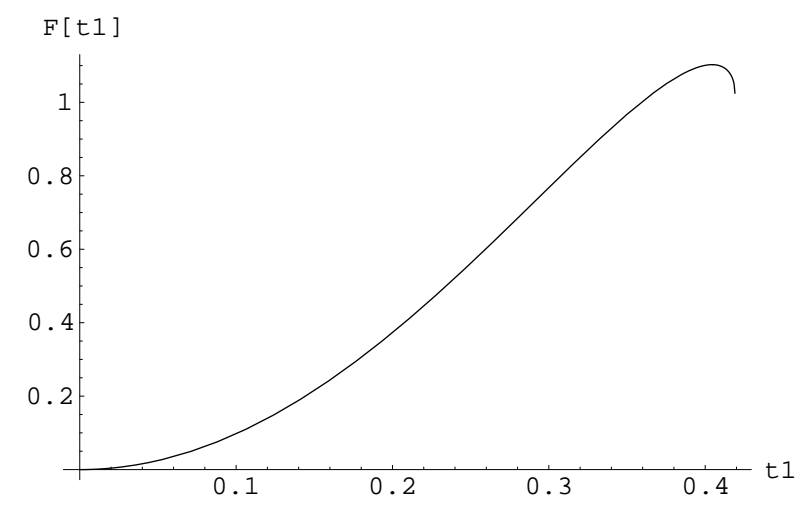

Figure 1: The plot of $\sin ^{2}(\pi \lambda)=F\left(t_{1}\right)$ vs. $t_{1}$ at level $(1,2)$ calculated using $(5.20)$.

Substituting the expression for $t_{0}$ from (5.19) at $R=1$ we get $F\left(t_{1}\right)$ as a function of $t_{1}$. In Fig. 1 we have displayed a plot of $F\left(t_{1}\right)$ vs. $t_{1}$ calculated using this formula. From this we see that the right hand side of (5.20) becomes larger than one even before $t_{1}$ reaches the critical point where the marginal and the vacuum branches meet, and hence $\lambda$ calculated from (5.6) becomes complex in this region. Since we argued earlier that $F\left(t_{1}\right)$ is bounded from above by 1 , we expect this to be an artifact of the level truncation approximation.

Calculation at higher level can be carried out using numerical methods. The full $R$ dependent tachyon potential needed for calculations up to level $(4,8)$ was given in $[6]$ (appendix B). With the help of this result and eq.(5.6) we can easily compute $F\left(t_{1}\right)$ as a function of $t_{1}$ at various levels of approximation up to level $(4,8)$. The results have been shown in Fig.2. In this diagram the graph which extends further to the right corresponds to result in a higher level approximation. We see from this graph that the result for $F\left(t_{1}\right)$ again exceeds unity beyond a certain value of $t_{1}$, and hence beyond this value the $\lambda$ defined through (5.6) becomes complex. This seems to be in conflict with the general result proved earlier that $F\left(t_{1}\right)$ is bounded from above by unity. We believe this is due to the fact that we have not reached a sufficiently high level of approximation. At least the results show that the growth of the maximum (which at level (4,8) is slightly above 1.2) slows down with increasing level of approximation. Thus it could turn around at higher level. Clearly we need explicit numerical results at higher level to settle this issue. The extrapolation metods of refs. [24, 25] may also be useful for this study.

Although we have not resolved the numerical problem, we have tried to isolate the 


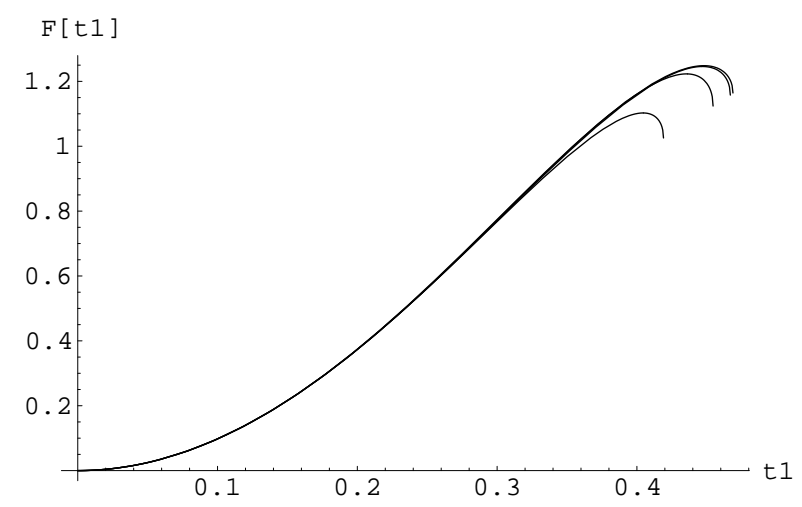

Figure 2: The plot of $\sin ^{2}(\pi \lambda)=F\left(t_{1}\right)$ vs. $t_{1}$ at level $(1,2),(2,4),(3,6)$ and $(4,8)$ approximation. The graph which extends further to the right corresponds to result in a higher level approximation. The level $(3,6)$ and $(4,8)$ results are almost indistinguishible in this graph. We see that the result exceeds unity beyond a certain value of $t_{1}$, and hence beyond this value the $\lambda$ defined through (5.6) becomes complex.

origin of the problem. From (5.6) we see that there are two possible sources of error. The $\mathcal{V}_{\text {eff }}\left(t_{1} ; 1\right)$ which is supposed to vanish identically is not zero at finite level, and the $2 \pi^{2} \mathcal{V}_{\text {eff }}^{\prime}\left(t_{1} ; 1\right)$ which is supposed to take the value -1 at its minimum, is not actually -1 at a finite level. It turns out that the contribution from the first term is negligible, and almost all the error comes from the second term. In particular, if we calculate $2 \pi^{2}\left(\mathcal{V}_{\text {eff }}\left(t_{1} ; R\right)-\mathcal{V}_{\text {eff }}\left(t_{1} ; 1\right)\right) /\left(R^{-1}-1\right)$, then according to the energy conjecture (4.8) and the vanishing of $\mathcal{V}_{\text {eff }}\left(t_{1} ; 1\right)$ this is supposed to take the value 1 at its maximum. Instead we find that for values of $R$ close to unity, this ratio is about 1.2 at its maximum in level $(4,8)$ approximation. If we believe that the energy conjecture (4.8) is satisfied, then this ratio must come down to unity at higher level approximation, and consequently the maximum value of $F\left(t_{1}\right)$ will also come down to unity.

One feature that we observe from Fig.2 is that the maximum of $F\left(t_{1}\right)$ is quite close to the critical value of $t_{1}$ where the level truncation method breaks down. Assuming that the maximum does come down to unity at higher level in agreement with the analytical result, it is tempting to speculate that the critical value of $t_{1}$ coincides with this maximum and hence describes the $\lambda=\frac{1}{2}$ point. Since the range $-\frac{1}{2} \leq \lambda \leq \frac{1}{2}$ can be thought of as the fundamental domain in the moduli space of lump solutions (with solutions 
outside this range being equivalent to the solutions in this range) this would imply that string field theory lump solutions precisely cover one fundamental domain. Only more detailed numerical analysis can tell us if this is so. However it is amusing to note that for superstring theory this is precisely what happens[26] for the periodic solutions of the effective action given in [27].

Acknowledgement: I wish to thank B. Zwiebach for useful discussions and many helpful comments on the manuscript.

\section{References}

[1] C. G. . Callan, I. R. Klebanov, A. W. W. Ludwig and J. M. Maldacena, Nucl. Phys. B 422, 417 (1994) [arXiv:hep-th/9402113].

[2] J. Polchinski and L. Thorlacius, Phys. Rev. D 50, 622 (1994) [arXiv:hep-th/9404008].

[3] A. Recknagel and V. Schomerus, Nucl. Phys. B 545, 233 (1999) [arXiv:hepth/9811237].

[4] A. Sen, JHEP 0204, 048 (2002) [arXiv:hep-th/0203211].

[5] A. Sen, JHEP 0207, 065 (2002) [arXiv:hep-th/0203265].

[6] A. Sen and B. Zwiebach, JHEP 0010, 009 (2000) [arXiv:hep-th/0007153].

[7] T. Takahashi and S. Tanimoto, Prog. Theor. Phys. 106, 863 (2001) [arXiv:hepth/0107046].

[8] M. Fujita and H. Hata, JHEP 0305, 043 (2003) [arXiv:hep-th/0304163].

[9] V. A. Kostelecky and S. Samuel, Nucl. Phys. B 336, 263 (1990).

[10] V. A. Kostelecky and S. Samuel, Nucl. Phys. B 336, 263 (1990).

[11] A. Sen and B. Zwiebach, JHEP 0003, 002 (2000) [arXiv:hep-th/9912249].

[12] N. Moeller and W. Taylor, Nucl. Phys. B 583, 105 (2000) [arXiv:hep-th/0002237].

[13] P. Di Vecchia, M. Frau, I. Pesando, S. Sciuto, A. Lerda and R. Russo, Nucl. Phys. B 507, 259 (1997) [arXiv:hep-th/9707068]. 
[14] E. Witten, Nucl. Phys. B268, 253 (1986).

[15] N. Moeller and B. Zwiebach, JHEP 0210, 034 (2002) [arXiv:hep-th/0207107].

[16] H. Yang, JHEP 0211, 007 (2002) [arXiv:hep-th/0209197].

[17] J. Gomis, K. Kamimura and J. Llosa, Phys. Rev. D 63, 045003 (2001) [arXiv:hepth/0006235].

[18] J. Gomis, K. Kamimura and T. Ramirez, arXiv:hep-th/0311184.

[19] L. Brekke, P. G. O. Freund, M. Olson and E. Witten, Nucl. Phys. B 302, 365 (1988).

[20] P. H. Frampton and Y. Okada, Phys. Rev. D 37, 3077 (1988).

[21] D. Ghoshal and A. Sen, Nucl. Phys. B 584, 300 (2000) [arXiv:hep-th/0003278].

[22] N. Moeller, A. Sen and B. Zwiebach, JHEP 0008, 039 (2000) [arXiv:hep-th/0005036].

[23] P. Mukhopadhyay and A. Sen, JHEP 0102, 017 (2001) [arXiv:hep-th/0101014].

[24] W. Taylor, JHEP 0303, 029 (2003) [arXiv:hep-th/0208149].

[25] D. Gaiotto and L. Rastelli, JHEP 0308, 048 (2003) [arXiv:hep-th/0211012].

[26] A. Sen, arXiv:hep-th/0312003.

[27] N. Lambert, H. Liu and J. Maldacena, arXiv:hep-th/0303139. 
